phys. stat. sol. (b) 228, No. 2, 617-619 (2001)

\title{
Electron Transport in III-V Nitride Two-Dimensional Electron Gases
}

\author{
D. Jena $\left.{ }^{1}\right)$ (a), I. Smorchkova (b), A. C. Gossard (a, c), and U. K. Mishra (a) \\ (a) Department of Electrical and Computer Engineering, University of California, \\ Santa Barbara, CA 93106, USA \\ (b) TRW, One Space Park R6/1563B, Redondo Beach, CA 90277, USA \\ (c) Materials Department, University of California, Santa Barbara, CA 93106, USA
}

(Received June 26, 2001; accepted July 4, 2001)

Subject classification: 72.20.Dp; 72.80.Ey; S7.14

We present a study of electron scattering processes in $\mathrm{AlGaN} / \mathrm{GaN}$ two-dimensional electron gases. A theoretical study of the effect of deformation potential scattering from strain fields surrounding dislocations is presented. The most important scattering mechanisms limiting electron transport are identified. We find that for $\mathrm{AlGaN} / \mathrm{GaN} 2 \mathrm{DEGs}$, mobility is limited by alloy scattering at high 2DEG densities. For AlN/GaN 2DEGs, interface roughness scattering limits mobility at high densities; there is a large improvement by the removal of the alloy barrier. At low 2DEG densities, dislocation scattering from charged cores and strain fields are the dominant scattering mechanisms.

Introduction The effect of dislocations on electron transport is generally assumed to occur by coulombic scattering from a charged core. Here, we show that deformation potential scattering from the strain fields surrounding dislocations is as important as the charged core in carrier scattering. To study the effect of strain field scattering on electron transport in $\mathrm{AlGaN} / \mathrm{GaN}$ 2DEGs, we evaluate the contribution of all scattering mechanisms to electron mobility.

Dislocation Scattering in 2DEGs The effect of dislocation scattering on electron transport in 2DEGs has been a relatively unexplored area. Recently, we derived the scattering rate for a $2 \mathrm{DEG}$ electron in the presence of a charged dislocation line [1]. In addition to the possibility of the core being charged, there exist strain fields around dislocations. These strain fields cause a perturbation of the potential for the effective mass electrons, and will cause scattering.

The strain field around an edge dislocation is given by

$$
u(r, \theta)=-\frac{b_{\mathrm{e}}}{2 \pi} \frac{1-2 \gamma}{1-\gamma} \frac{\sin \theta}{r} .
$$

The strain field leads to a compression/dilatation of the unit cells around the dislocation. The deformation potential theorem of Bardeen and Shockley tells us that the shift in the conduction band edge due to this is given by

$$
\Delta E_{\mathrm{C}}(r, \theta)=\Xi_{i j} \operatorname{Tr}\left[u_{i j}\right]=-\frac{b_{\mathrm{e}} \Xi_{\mathrm{d}}^{\mathrm{CB}}}{2 \pi} \frac{1-2 \gamma}{1-\gamma} \frac{\sin \theta}{r},
$$

\footnotetext{
1) Corresponding author; Phone: +1 805893 5404; Fax: +1 805893 5714; e-mail: djena@engineering.ucsb.edu
} 
where $\Xi_{i j}$ are the components of the conduction band deformation potential tensor in GaN. For the conduction band (CB) perturbation, the $\mathrm{CB}$ deformation potential $\Xi_{\mathrm{d}}^{\mathrm{CB}}$ at the $\Gamma$-point of bandstructure is used. Using this as the scattering potential, we derive the momentum scattering rate for 2DEG electrons in the Born approximation, using Fermi's golden rule. We derive the $2 \mathrm{DEG}$ electron mobility limited by strain field scattering of threading edge dislocations to be

$$
\mu_{\mathrm{disl}}^{\text {strain }}=\frac{2 e \hbar^{3} \pi k_{\mathrm{F}}^{2}}{N_{\mathrm{disl}} m^{* 2} b_{\mathrm{e}}^{2} \Xi^{2}}\left(\frac{1-\gamma}{1-2 \gamma}\right)^{2} \frac{1}{I_{\mathrm{disl}}^{\text {strain }}\left(n_{\mathrm{s}}\right)} .
$$

where $\hbar$ is the reduced Planck constant, $k_{\mathrm{F}}=\sqrt{2 \pi n_{\mathrm{s}}}$ is the Fermi wavevector for the 2DEG, $N_{\text {disl }}$ is the dislocation density in $\mathrm{cm}^{-2}, \gamma$ is the Poisson ratio for the crystal, $m^{*}$ is the conduction band effective mass, $\Xi_{\mathrm{d}}^{\mathrm{CB}}=-8.0 \mathrm{eV}$ is the conduction band deformation potential, and $b_{\mathrm{e}}$ is the Burgers vector for the dislocation. $I_{\text {disl }}^{\text {strain }}\left(n_{\mathrm{s}}\right)$ is a dimensionless integral depending only on the $2 \mathrm{DEG}$ carrier density ${ }^{2}$ ).

All Scattering Mechanisms How strong is the effect of deformation potential scattering from the strain fields around dislocations? To evaluate this, we calculate the effect of all scattering processes affecting electron mobility at low temperatures. We consider scattering by background unintentional donors $\left(N_{\text {back }} \approx 10^{17} \mathrm{~cm}^{-3}\right)$, scattering by the alloy disorder due to finite penetration of the wavefunction into the alloy AlGaN barrier (this is absent for $\mathrm{AlN}$ barriers), interface roughness at the $\mathrm{Al}(\mathrm{Ga}) \mathrm{N} / \mathrm{GaN}$ heterojunction $(\Delta=2.5 \AA, L=10 \AA)$, scattering from remote surface donors $\left(N_{\text {surf }} \approx n_{\mathrm{s}}\right)$, acoustic phonon scattering, and dislocation scattering.

Low temperature mobility reveals important facts about impurities in the heterostructure and gives valuable clues for the design of better devices. We plot the calculated low temperature 'mobility map' against 2DEG sheet density in Fig. 1. The highest reported experimental electron mobilities [2] are also plotted in the same figure for both alloy (AlGaN) and binary (AlN) barriers.

From the plot, it is clear that alloy scattering is severe, and is the main low temperature mobility limiting scattering mechanism. At high carrier densities $\left(n_{\mathrm{s}} \geq 10^{13} \mathrm{~cm}^{-2}\right)$, it starts affecting even room temperature electron transport. The reason is that electron mobility limited by polar optical phonon scattering at room temperature is $\mu_{300 \mathrm{~K}}^{2 \mathrm{DEG}} \approx 2000 \mathrm{~cm}^{2} / \mathrm{V} \mathrm{s}$, and as is evident from Fig. 1 , alloy scattering reaches that value when $n_{\mathrm{s}}$ exceeds $10^{13} \mathrm{~cm}^{2}$.

Removal of the alloy barrier by growing thin AlN layers causes a marked jump in the mobility for high sheet densities [3]. This is depicted by a jump from the curve of alloy scattering limited mobility to interface roughness limited mobility. In Fig. 1a, the empty circles represent data for AlGaN barriers and the filled circles for AlN barriers. Since this translates to a higher conductivity, introduction of a thin AlN layer at the $\mathrm{AlGaN} / \mathrm{GaN}$ interface is an attractive technique for high-electron mobility transistors.

As is evident, dislocation scattering dominates electron transport properties at low sheet densities. Figure $1 \mathrm{~b}$ shows the effect of different dislocation densities on maximum achievable electron mobilities. Note that deformation potential scattering from

2) $I_{\mathrm{disl}}^{\mathrm{strain}}\left(n_{\mathrm{s}}\right)=\int_{0}^{1} \frac{u^{2} \mathrm{~d} u}{\left(u+q_{\mathrm{TF}} / 2 k_{\mathrm{F}}\right)^{2} \sqrt{1-u^{2}}}$, where $q_{\mathrm{TF}}$ is the Thomas-Fermi wavevector for 2DEGs, and $k_{\mathrm{F}}=\sqrt{2 \pi n_{\mathrm{s}}}$ is the Fermi wavevector. 

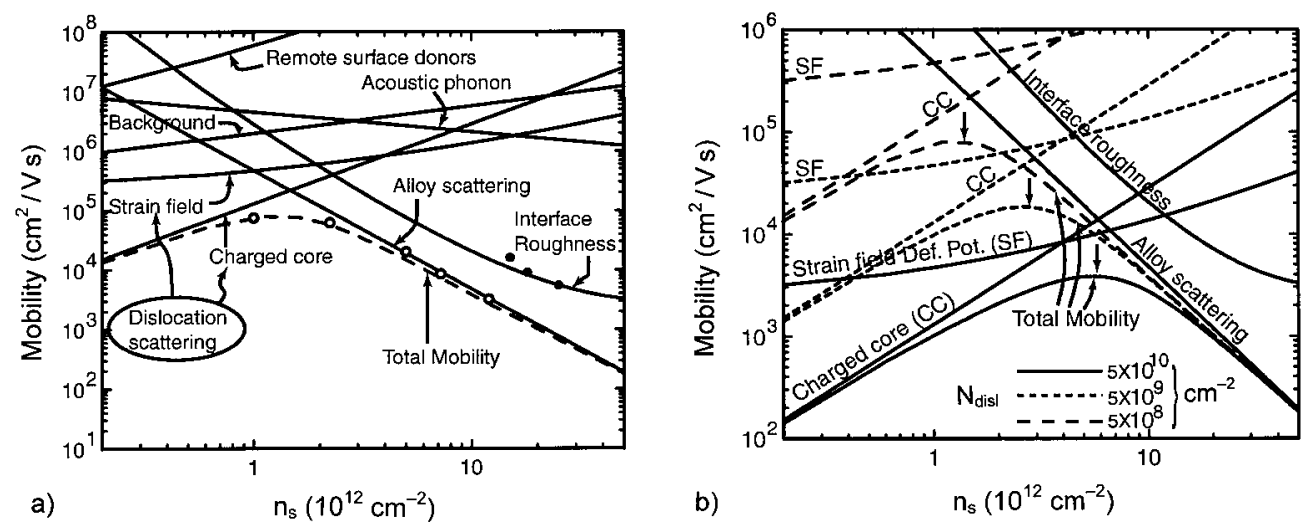

Fig. 1. a) Scattering processes limiting electron mobility in the $\mathrm{Al}(\mathrm{Ga}) \mathrm{N} / \mathrm{GaN} 2 \mathrm{DEG}$ system evaluated for $N_{\text {disl }}=5 \times 10^{8} \mathrm{~cm}^{-2}$; experimental data is also shown (circles). b) Effect of dislocation scattering for three different dislocation densities

the strain fields of dislocations is as important as charged core scattering. Inverted arrows show the peak mobilities achievable for the respective dislocation densities.

In conclusion, we studied the effect of strain fields or dislocations on electron transport properties. We have identified the chief mobility limiting scattering mechanisms for various $2 \mathrm{DEG}$ densities. The identification of the chief scattering process in HEMTs has led us to propose a method for an improvement of conductivity.

\section{References}

[1] D. Jena, A.C. Gossard, and U.K. Mishra, Appl. Phys. Lett. 76, 1707 (2000).

[2] I. P. SmorchKova et al., J. Appl. Phys. 86, 4520 (1999).

[3] I. P. Smorchkova et al., Appl. Phys. Lett. 77, 3998 (2000). 